\title{
Q fever: person to person transmission within a family
}

\author{
J S MANN, J G DOUGLAS, J M INGLIS, A G LEITCH \\ From the Chest Unit and Regional Virus Laboratory, City Hospital, Edinburgh
}

$\mathrm{Q}$ fever is caused by a rickettsia like organism, Coxiella burnetii, and is responsible for $1 \%$ of all hospital admissions with pneumonia in Great Britain. ${ }^{1}$ Infection in man is most commonly acquired by exposure to domestic animal carriers, by contact with dust or straw, or by drinking unpasteurised milk contaminated with the organism. ${ }^{2}$ Previous reports suggest that case to case transmission in man is rare. ${ }^{3-6}$ We report the apparent person to person transmission, within a family, of $C$ burnetii infection originating from a veterinary sheep station.

\section{Case report}

A 79 year old man (case 5-figure) was admitted with a 24 hour history of sharp chest pain, breathlessness, vomiting, and incontinence of urine. Before this illness the patient had been largely confined to his home as a result of Parkinson's disease and a previous stroke. On examination he was febrile and tachypnoeic and a few basal inspiratory crackles were audible on auscultation. The chest radiograph showed no abnormality on admission to hospital but subsequent films showed the development of right upper lobe consolidation. He was treated with erythromycin $500 \mathrm{mg}$ four times daily, after which his condition improved and the chest radiographic appearances returned to normal. A diagnosis of recent $Q$ fever infection was subsequently made when complement fixation testing indicated $C$ burnetii antibody titres of less than 16 for phase I and 512 for phase II.

Five weeks before his admission the patient's wife (case 4) had been treated at home (house 2) for pneumonia. Throughout her illness she had noted symptoms of dyspnoea, chest tightness, and confusion. She was subsequently found to have serological evidence of recent $Q$ fever infection, with a phase I titre of 16 and a phase II titre of 51218 weeks after her illness. Neither of these two patients had had any contact with animals.

Further inquiry revealed that the patient's married daughter (case 3), who lived six miles away (house 1), had had a similar illness seven weeks before that of her mother; 29 weeks after this illness she had serological evidence of recent C burnetii infection, with a phase II titre greater than 256 . The patient's grand daughter (case 2), who lived with her parents, had suffered a similar illness a week before her mother, but unfortunately no specimen could be obtained for serological testing. Further inquiries revealed that the son in law (case 1), who had remained well, was employed as

Address for reprint requests: Dr JS Mann, Department of Respiratory Medicine, City General Hospital, Stoke on Trent.

Accepted 3 June 1986

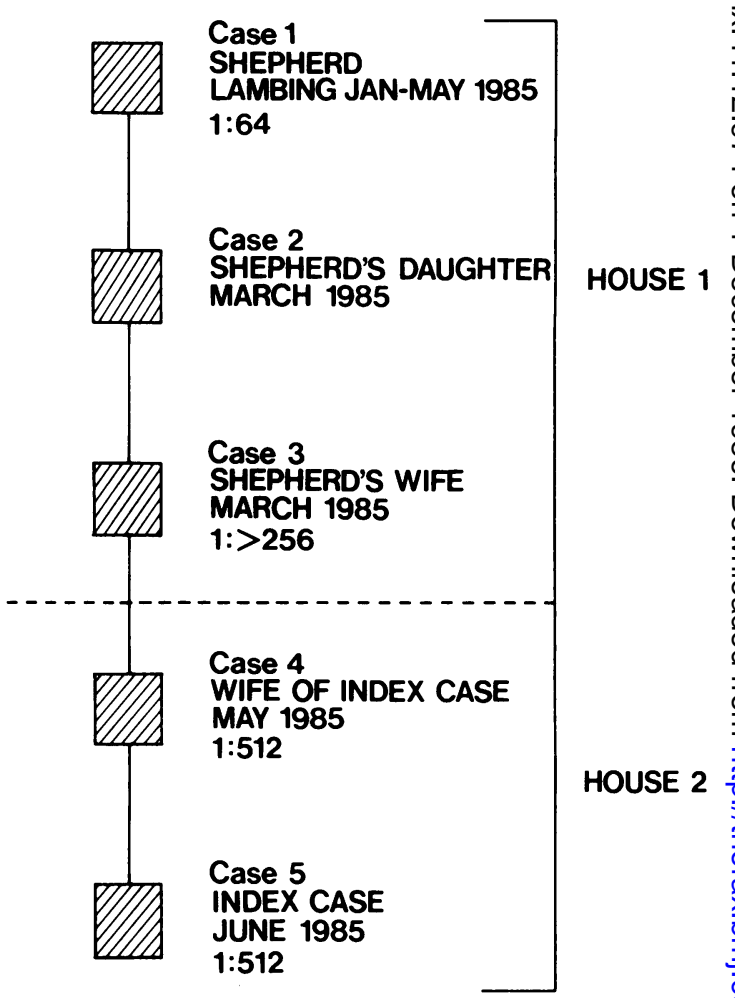

Proposed epidemiological pathway of infection, showing dates of illnesses and titres of complement fixing antibody to Coxiella burnetii phase II antigen.

a shepherd at a local veterinary sheep station and had been lambing during the period when his wife and daughter had become ill. He also had evidence of recent $C$ burnetii infection with a phase II titre of 64 . It is pertinent that he worked in overalls that were always washed at home and that he changed before visiting his wife's parents' house. Ailing animals were never brought to the house and none of the family drank unpasteurised milk.

\section{Discussion}

$Q$ fever was first described by Derrick ${ }^{7}$ in a group of abattoir workers in Queensland, Australia, in 1937. It is caused by a rickettsia like organism, now termed Coxiella burnetii. This organism most commonly affects the genital tract of wild and domestic animals, including cows, sheep, and goats, but 
rarely causes disease. Spread to man occurs usually by inhalation of the organism. After an incubation period of 14-28 days patients may develop an acute illness with chills and sweating associated with areas of pulmonary consolidation on the chest radiograph. ${ }^{2}$ Raised complement fixing antibody titres to the phase II antigen may provide evidence of acute infection. High titres to phase I antigen are found in chronic infections such as endocarditis. Infection may also arise in man after ingestion of $C$ burnetii through drinking unpasteurised milk and, rarely, after a contaminated blood transfusion. ${ }^{8}$ Case to case transmission of $C$ burnetii in man is rare ${ }^{3-6}$ and only two such reports have been published in English ${ }^{34}$ In one case staff in a hospital were infected by a patient ${ }^{4}$ and in the other pathologists and a mortuary technician were infected during a postmortem examination. ${ }^{3}$

We believe that our cases provide a rare example of person to person transmission of $C$ burnetii infection within a family. The son in law (case 1) was probably infected during the lambing season, since the organism may be shed in large numbers at parturition. Infection has been reported after contact with contaminated clothing, ${ }^{9}$ and as the shepherd went home in his working clothes possibly infection of his daughter (case 2) and wife (case 3) occurred by this route. Alternatively, however, the shepherd may have transmitted the organism himself since on the evidence of a raised antibody titre he had an asymptomatic infection with the organism. Transfer of the organism on clothing could not account for the subsequent infection of the shepherd's relatives, who lived several miles away, as he invariably changed from his working clothes before visiting them. In addition, the time course of the illnesses is suggestive of sequential spread of infection. We are unaware of any other cases of $Q$ fever in the vicinity of cases 4 and 5 during the time of their illnesses and the regional virus laboratory received no sera positive for $C$ burnetii from the area around the household during the first six months of 1985 . The homes of the families were situated in semirural areas not associated with a high density of domestic animals, making infection from another animal source unlikely.

For these reasons we believe that this report provides the first example of person to person transmission of $C$ burnetii in a family. It also emphasises the need to consider this organism as a cause of pneumonia in what may initially appear to be the unlikeliest of cases.

We wish to thank Miss Joyce Holywell for typing the manuscript.

\section{References}

1 Pugh S. British Thoracic Society Pneumonia study: Microbiological results. [abstract]. Thorax 1985;40: 693-4.

2 Christie AB. Q fever. In: Christie AB, ed. Infectious diseases, epidemiology, and clinical practice. Edinburgh: Churchill Livingstone, 1980:800-12.

3 Harman JB. Q fever in Great Britain. Clinical account of eight cases. Lancet 1949;ii:1028-30.

4 Deutch DL, Peterson ET. Q fever: transmission from one human being to others. JAMA 1950;143:348-50.

5 Siegert R, Simrock W, Stroeder U. Ueber einen epidemischen Ausbruch van Q-Fieber in einen Krankenhause. $Z$ Parasitol 1950;2:1-40.

6 Trub CLP, Boese W, Posch J. Die Q-Fieber-Epidemia am Niederrhein 1958. Arch Hyg Bakteriol 1960;144: 48-73.

7 Derrick EH. "Q" fever, a new fever entity: clinical features, diagnosis and laboratory investigations. Med J Aust 1937;ii:281-99.

8 Warren JW, Hornick RB. Coxiella burnetii. In: Mandell GL, Douglas RG, Bennett JE, eds. Principles and practice of infectious diseases. New York: John Wiley, 1979:1516-20.

9 Stoker MGP, Marmion BP. The spread of $Q$ fever from animals to man. The natural history of a rickettsial disease. Bull World Health Org 1955;13:781-806. 